\title{
Quantitative proteomics reveals divergent responses in Apis mellifera worker and drone pupae to parasitization by Varroa destructor
}

\author{
Carla Surlis $^{\mathrm{a}}$, James C. Carolan ${ }^{\mathrm{a}}$, Mary Coffey ${ }^{\mathrm{b}}$, Kevin Kavanagh ${ }^{\mathrm{a}, *}$ \\ a Department of Biology, Maynooth University, Maynooth, Co. Kildare, Ireland \\ ${ }^{\mathrm{b}}$ Department of Life Sciences, University of Limerick, Limerick, Ireland
}

\section{A R T I C L E I N F O}

\section{Keywords:}

Apis mellifera

Immune response

Mite

Parasite

Proteomics

Varroa destructor

\begin{abstract}
A B S T R A C T
Varroa destructor is a haemophagous ectoparasite of honeybees and is considered a major causal agent of colony losses in Europe and North America. Although originating in Eastern Asia where it parasitizes Apis cerana, it has shifted hosts to the western honeybee Apis mellifera on which it has a greater deleterious effect on the individual and colony level. To investigate this important host-parasite interaction and to determine whether Varroa causes different effects on different castes we conducted a label free quantitative proteomic analysis of Varroa-parasitized and non-parasitized drone and worker Apis mellifera pupae. 1195 proteins were identified in total, of which 202 and 250 were differentially abundant in parasitized drone and worker pupae, respectively. Both parasitized drone and worker pupae displayed reduced abundance in proteins associated with the cuticle, lipid transport and innate immunity. Proteins involved in metabolic processes were more abundant in both parasitized castes although the response in workers was more pronounced. A number of caste specific responses were observed including differential abundance of numerous cytoskeletal and muscle proteins, which were of higher abundance in parasitized drones in comparison to parasitized workers. Proteins involved in fatty acid and carbohydrate metabolism were more abundant in parasitized workers as were a large number of ribosomal proteins highlighting either potentially divergent responses to Varroa or a different strategy by the mite when parasitizing the different castes. This data improves our understanding of this interaction and may provide a basis for future studies into improvements to therapy and control of Varroasis.
\end{abstract}

\section{Introduction}

Varroa destructor is an ectoparasite of the western honeybee, Apis mellifera, and its deleterious effects have been responsible for large scale losses in both natural and commercial settings. Varroa is a completely host-dependent mite, lacking a free living stage with its life cycle completed within the bee brood cells. Varroa feed communally and repeatedly on developing larvae and pupae leading to the spread of pathogens within colonies (Parker et al., 2012). Parasitization by Varroa has a serious effect on the health of the bee through loss of haemolymph, transmission of viruses and other microbes, and by causing a reduction in body weight (Bowen-Walker and Gunn, 2001, Levin et al., 2016, Wilfert et al., 2016).

Although originating in Eastern Asia where it parasitizes Apis cerana, it has shifted hosts to the western honeybee Apis mellifera on which it has a greater deleterious effect on individual and colony level fitness (Chen et al., 2017, Beaurepaire et al., 2015). The lack of a definite preference by Varroa for drone brood during host finding in $A$. mellifera colonies and the poor ability of $A$. mellifera to dislodge feeding Varroa during grooming contribute to much higher infestation levels in A. mellifera colonies than in Apis cerana which was the original host of V. destructor (Büchler et al., 1992, Fries et al., 2006, Parker et al., 2012). Worker bees are the largest caste in the colony and are essential to the survival of the colony, and the lack of drone brood preference by Varroa in A. mellifera contributes to their negative effect on colonies (Parker et al., 2012). Varroa have adversely affected apiculture in every country where present, leading to the loss of hundreds of thousands of colonies (Zakar et al., 2014, Kuster et al., 2014). Given the importance of Apis mellifera in honey production and a provider of key ecosystem and agricultural services such as plant pollination, it is surprising how little is known about this host-parasite interaction at the molecular level. To address this a label free quantitative proteomic analysis of Varroaparasitized and non- parasitized drone and worker pupae was conducted.

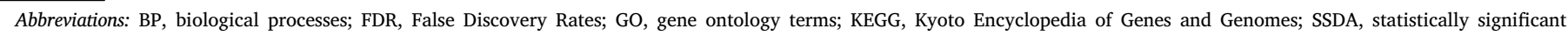
differentially abundant; MP, molecular function; SSDA, statistically significant differentially abundant

* Corresponding author.

E-mail address: kevin.kavanagh@nuim.ie (K. Kavanagh). 
Recent advances in proteomic and genomic technologies has resulted in significant insights into honeybee biology and the Apis-Varroa interaction at the molecular level (Qi et al., 2015). High-resolution quantitative mass spectrometry (MS) routinely quantify and identify thousands of proteins across multiple samples in a single run which gives an unprecedented opportunity to examine changes in proteomic profile of a particular biological fraction or organism. We previously utilised this technology to study the mechanisms conferring resistance to Bayvarol in $V$. destructor and the results indicated a decreased abundance of cuticle associated proteins and an increase in the abundance of proteins involved in cellular detoxification as possible factors in the resistant phenotype (Surlis et al., 2016). Similar proteome-wide analysis has been utilised to investigate the molecular determinants of hygienic behaviours and resistance/sensitivity to $V$. destructor (Parker et al., 2012; Hu et al., 2016). Proteomics has also been used to investigate developmental processes and differentiation of drone and worker bees (Fang et al., 2014, Fang et al., 2015) and the phosphoproteome of the hypopharyngeal gland in A. mellifera worker bees (Qi et al., 2015) has also been reported.

In the work presented here label free quantitative (LFQ) mass spectrometry was utilised to compare the proteomes of Varroa-parasitized and non-parasitized drone and worker A. mellifera pupae to gain insight into the interaction between the mite and honeybee host.

\section{Materials and methods}

\subsection{Mellifera pupae collection}

A. mellifera purple-eye stage pupae were collected from three separate colonies within an apiary in Kilmessan, County Meath, Ireland (coordinates 53.558980, -6.660039). For the drone collection, brood boards were removed from hives, stored in insulated boxes and transported to the laboratory where they were stored at $-80^{\circ} \mathrm{C}$ prior to protein extraction from drone pupae. Time from removal of brood boards from hive to freezing in laboratory in $<1 \mathrm{~h}$. Following removal of the drone boards, a queen trapping method was used to concentrate Varroa within worker brood cells by placing a queen excluder on a single brood frame, and moving to a new board every week following removal of the worker brood. Boards were stored at $-80^{\circ} \mathrm{C}$ for subsequent worker brood protein extraction and analysis. To avoid variability between samples pupae were chosen at the purple eye stage with no darkening of the cuticle. Capped drone and worker cells were opened and pupae were inspected for Varroa and where present, the mature adult female and immature mites were enumerated. Pupae from cells containing 4 adult female mites and 8 immature offspring were considered heavily parasitized and were chosen for subsequent analysis. Pupae from three different colonies were collected, mixed and randomly chosen. Four independent biological replicates were used for the analysis. Pupae were not screened for the presence of viruses or bacteria.

\subsection{Sample preparation and mass spectrometry}

Individual parasitized or unparasitized pupae (number of replicates $=4$ ) were homogenized using a pellet pestle in $7 \mathrm{M}$ urea; $2 \mathrm{M}$ thiourea buffer supplemented with a protease inhibitor cocktail tablet (Complete $^{\mathrm{TM}}$ Protease Inhibitor Cocktail (Roche)). Samples were centrifuged at $9000 \mathrm{~g}$ for $5 \mathrm{~min}$, the lipid layer was removed using a spatula, and the supernatant transferred to a fresh tube. Proteins were quantified by Bradford assay and $300 \mu \mathrm{g}$ of protein was removed and acetone precipitated overnight at $-20^{\circ} \mathrm{C}$. Proteins were centrifuged at $10,000 \mathrm{~g}$ for $10 \mathrm{~min}$ and the pellet resuspended in $300 \mu \mathrm{l}$ of $8 \mathrm{M}$ urea. Samples were re-quantified using Qubit Fluorometer (Invitrogen), $75 \mu \mathrm{g}$ was removed, reduced with dithiotreitol (DTT; $200 \mathrm{mM}$ ), alkylated with iodoacetamide (IAA; $1 \mathrm{M}$ ) and digested with sequence grade trypsin (Promega) at a trypsin:protein ratio of $1: 40$, overnight at $37^{\circ} \mathrm{C}$. Tryptic peptides were purified for mass spectrometry using C18 spin filters
(Medical Supply Company) and $0.70 \mu$ g of the peptide mix was eluted onto a QExactive (ThermoFisher Scientific, U.S.A) high resolution accurate mass spectrometer connected to a Dionex Ultimate 3000 (RSLCnano) chromatography system. Peptides were separated by an increasing acetonitrile gradient on a Biobasic C18 PicofritTM column (100 mm length, $75 \mathrm{~mm} \mathrm{ID),} \mathrm{using} \mathrm{a} 120 \mathrm{~min}$ reverse phase gradient at a flow rate of $250 \mathrm{~nL} / \mathrm{min}$. All data were acquired with the mass spectrometer operating in automatic data dependent switching mode. A high-resolution MS scan (300-2000 Dalton) was performed using the Orbitrap to select the 15 most intense ions prior to MS/MS.

\subsection{Data and bioinformatic analysis}

Protein identification from the MS/MS data was performed using the Andromeda search engine in MaxQuant (version 1.2.2.5; http:// maxquant.org/) to correlate the data against the predicted protein set derived from the A. mellifera genome (Amel_4.5 assembly, Honeybee Genome sequence consortium, 2006). The following search parameters were used: first search peptide tolerance of $20 \mathrm{ppm}$, second search peptide tolerance $4.5 \mathrm{ppm}$ with cysteine carbamidomethylation as a fixed modification and $\mathrm{N}$-acetylation of protein and oxidation of methionine as variable modifications and a maximum of 2 missed cleavage sites allowed, all standard (Michalski et al., 2011). False Discovery Rates (FDR) were set to $1 \%$ for both peptides and proteins and the FDR was estimated following searches against a target-decoy database. Peptides with minimum length of seven amino acid length were considered for identification and proteins were only considered identified when more than one unique peptide for each protein was observed.

Results processing, statistical analyses and graphics generation were conducted using Perseus v.1.5.0.31 (www.maxquant.org). LFQ intensities were $\log 2$-transformed and ANOVA of significance and $t$-tests between the parasitized and non-parasitized pupae proteomes were performed using a p-value cut-off of 0.05 to identify statistically significant differentially abundant (SSDA) proteins. Proteins that had intensity values of zero (indicative of absence or very low abundance in a sample) were included in the study only when they were completely absent from one group and present in at least three of the replicates in the second group. These proteins were also included in the statistical analysis after imputation of representative numbers based on the lowest value for each data set, which was calculated as a 1.75 downshift from the mean value, allowing for 0.25 width in the downshift for standard deviation. Volcano plots were generated in Perseus to visualize differentially abundant proteins between control and parasitized groups and a principal component analysis (PCA) was performed using the normalized intensity values. Hierarchical clustering was performed on Z-score normalized intensity values for all differentially abundant proteins by clustering both samples and proteins using Euclidean distance and complete linkage.

The Uniprot identifier for each protein was obtained by conducting a BLAST search of all proteins identified against the Uniprot sequence set for Apis mellifera (WWW.Uniprot.org downloaded April 2017). The top Uniprot match was obtained for each identified protein and used to query the Perseus annotation file (downloaded April 2017) and extract terms for biological process, molecular function, Kyoto Encyclopaedia of Genes and Genomes (KEGG) name, KEGG pathway, protein family (pfam) and InterPro. The 'categories' function in Perseus was utilised to highlight and visualize the distribution of various pathways and processes on volcano plots. GO and KEGG term enrichment analysis was performed on the major protein clusters identified by hierarchical clustering using a Fisher's exact test (a Benjamini-Hochberg corrected FDR of 2\%) for enrichment in Uniprot Keywords, gene ontology biological process (GOBP), gene ontology cellular component (GOCC) and KEGG (FDR $<2 \%$ ).

The Search Tool for the Retrieval of Interacting Genes/Proteins (STRING) v10 (http://string-db.org/) was used to map known and predicted protein: protein interactions and to identify groups of proteins associated with specific pathways and processes. FASTA files were generated 
A

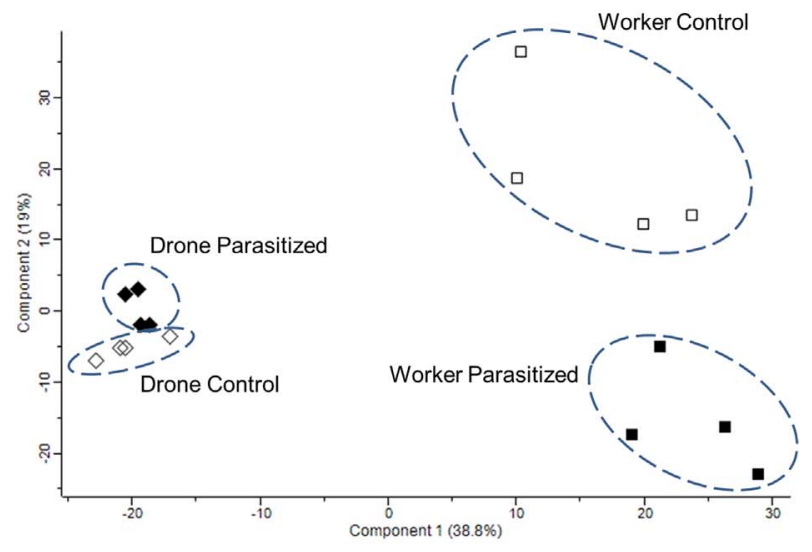

B

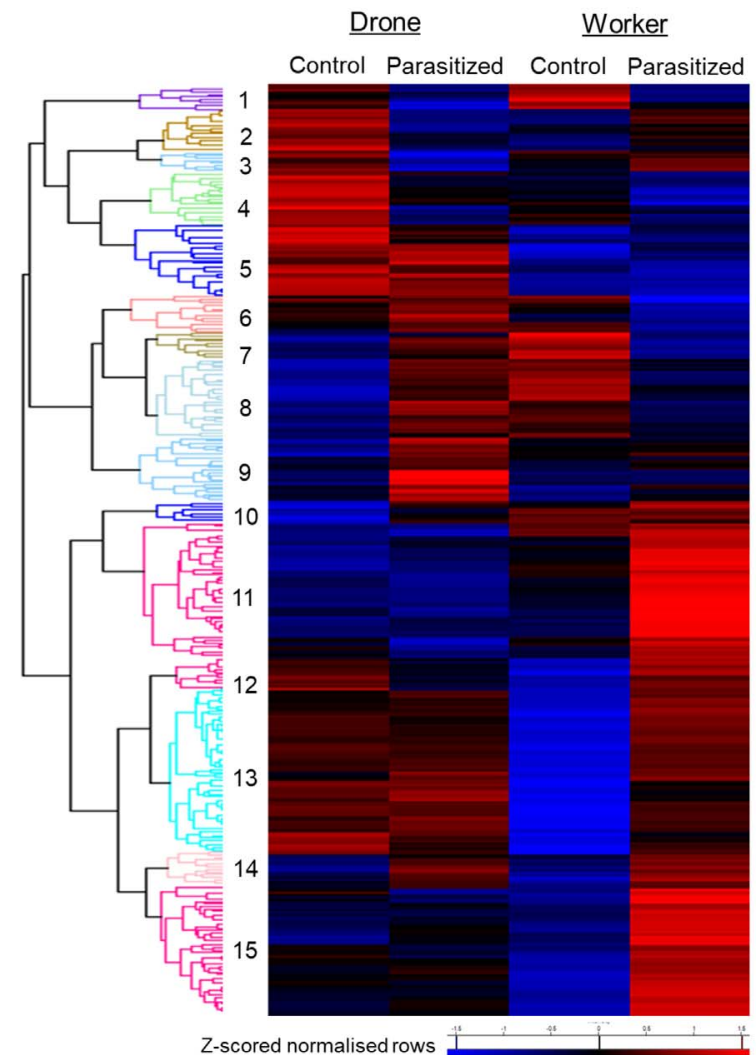

Fig. 1. Label free quantitative proteomic analysis of parasitized and non-parasitized worker and drone pupae. (A) Principal component analysis (PCA) of parasitized and nonparasitized worker and drone pupae. A clear distinction can be observed between the castes and in the extent of response to parasitization. (B) Hierarchical clustering (columns) and intensity heat map of mean protein expression values of all statistically significant differentially abundant proteins. 15 distinct groups of proteins were resolved.

of SSDA proteins found in each sample in Bioedit version 7.2.5.0 and inputted and analyzed in STRING using the medium to high confidence (0.5-0.7). GO term enrichment analyses for biological process, molecular function and cellular compartment were then conducted to identify associated pathways and processes. Pathways were examined using the KEGG pathway analysis (http://www.kegg.jp/kegg/tool/map_pathway2.html), using the 'KEGG Mapper-Search\&Color Pathway' tool.

\section{Results}

In order to characterize the effect of parasitization by Varroa on the developing pupa, the proteomic profiles of unparasitized worker and drone pupae were compared to those of heavily parasitized pupae using label free quantitative (LFQ) proteomics. Whole body protein extractions were performed on four replicates per sample group and subjected to mass spectrometry. In total, 27,305 peptides originating from 1195 proteins (Supplementary Table 1) were identified in drone and worker pupae post filtering. PCA (Fig. 1A) resolved the replicates of each sample and highlights a clear divergence in the proteomes of drone and worker pupae. In addition a greater difference in the proteomes of parasitized and unparasitized workers is observed in comparison to parasitized and unparasitized drones.

Forty-five and six proteins were deemed exclusive to parasitized and control worker samples respectively (Supplementary Table 1). Of the 45 proteins exclusively detected in parasitized workers in comparison to their controls, 18 are involved in metabolism, specifically, fatty acid and lipid metabolism (XP_006567467.1 fatty acid synthase; XP_006563706.1 3-hydroxyisobutyryl-CoA hydrolase; XP_394948.5 carnitine O-palmitoyltransferase 2; XP_001120311.1 trans-2,3-enoylCoA reductase-like and NP_001229455.1 fatty acyl-CoA reductase), amino acid degradation (XP_006566117.1 isovaleryl-CoA dehydrogenase; XP_392483.3 4-aminobutyrate aminotransferase and XP_006563706.1 3-hydroxyisobutyryl-CoA hydrolase) and aminoacyltRNA biosynthesis (XP_623880.1| PREDICTED: probable glutaminetRNA ligase, XP_624023.2 isoleucine-tRNA ligase and XP_006569450.1 and phenylalanine-tRNA ligase). Eleven of the 45 proteins were annotated as "mitochondrial" or "mitochondrial-like" indicating their association with mitochondria. Six proteins were detected only in nonparasitized workers in comparison to their parasitized counterparts and include two cuticle proteins (XP_006568331.1 cuticle protein 18.7; NP_001257759.1 cuticular protein 28 precursor), prefoldin subunit 2 (XP_006561235.1), isocitrate dehydrogenase subunit beta (XP_624511.1), nucleoprotein TPR (XP_006564561.1) and an uncharacterized protein LOC551742 (XP_624132.1).

Ten proteins were detected exclusively in both parasitized and nonparasitized drones when compared to each other. Proteins found only in parasitized drones included tubulin (XP_006563567.1), a leucine-rich repeat-protein (XP_006559699.1), MD-2-related lipid-recognition protein (XP_392711.3), long-chain-fatty-acid-CoA ligase (XP_006561674.1), GDP-L-fucose synthase (XP_006566989.1), gamma-glutamylcyclotransferase (XP_006563149.1), two mitochondrial associated proteins: hexaprenyldihydroxybenzoate methyltransferase (XP_395548.2) and 1-2hydroxyglutarate dehydrogenase (XP_006567228.1) and two uncharacterized proteins: LOC102654380 (XP_006571092.1) and LOC100577080 (XP_006561488.1). The ten proteins that were detected in all replicates of non-parasitized drones and absent in all replicates of parasitized drones were two zinc finger protein 512B proteins (XP_006562940.1 and XP_001121697.2), mitochondrial import receptor subunit TOM40 (XP_391836.2), slit homolog 2 (XP_397526.2), sideroflexin-3 (XP_392085.2), synaptobrevin homolog YKT6 (XP_624934.1), maternal protein exuperantia isoform (XP_006560074.1), U6 snRNA-associated Sm-like protein (XP_001120188.1), scavenger receptor class B protein (XP_392752.3) and UPF0160 protein MYG1 (XP_006568327.1).

Two sample t-tests $(\mathrm{p}<0.05)$ identified 202 and 250 SSDA proteins between unparasitized and parasitized worker pupae and unparasitized and parasitized drone pupae respectively (Supplementary Tables $1 \mathrm{c}$ and d) and the 30 most differentially abundant proteins for both groups are displayed in Table 1. The most abundant proteins in parasitized workers in comparison to the unparasitized controls were an uncharacterized protein (XP_003249952.2) with a relative fold change (RFC) of 9.9, a dolichyl-diphosphooligosaccharide glycosyltransferase (NP_001229451.1; RFC: 5.8); a histone H3 protein (XP_006570899.1; RFC: 5.3); hydroxyacid-oxoacid transhydrogenase (XP_003250818.2; RFC: 5.6) and glycerol-3-phosphate dehydrogenase (NP_001014994.1; RFC: 4). Proteins of highest abundance in non-parasitized workers in comparison to their parasitized counterparts included a myosin regulatory light chain protein (XP_006558384.1; 16.5 RFC), tropomyosin-1 (XP_006560146.1; 9.6 RFC), cuticular protein 22 (NP_001257743.1; 6.5 $\mathrm{RFC}$ ), an endocuticle structural glycoprotein (XP_001120797.3; 6.5 
Table 1

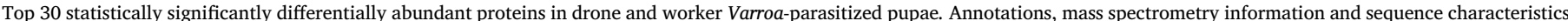

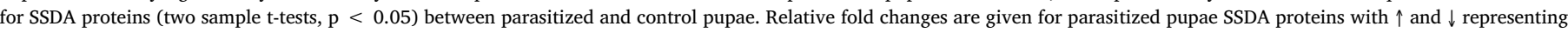
higher or lower abundance relative to the controls.

\begin{tabular}{|c|c|c|c|c|c|c|c|c|}
\hline Caste & Protein ID & Protein Annotation & Relative FC & Intensity & Peptides & $\begin{array}{l}\text { Sequence } \\
\text { coverage [\%] }\end{array}$ & $\begin{array}{l}\text { Mol. weight } \\
{[\mathrm{kDa}]}\end{array}$ & $\begin{array}{l}\text { MS/MS } \\
\text { Count }\end{array}$ \\
\hline \multirow[t]{30}{*}{ Worker } & XP_003249952.2 & Uncharacterized protein LOC100576161 & $10.0 \uparrow$ & $9.6 \times 10^{9}$ & 1 & 26.9 & 15.7 & 49 \\
\hline & NP_001229451.1 & Dolichyl-diphosphooligosaccharide glycosyltransferase & $5.8 \uparrow$ & $5.9 \times 10^{9}$ & 39 & 68.1 & 70.5 & 286 \\
\hline & XP_006570899.1 & Histone H3-like & $5.3 \uparrow$ & $4.5 \times 10^{9}$ & 9 & 60.3 & 15.4 & 65 \\
\hline & XP_003250818.2 & Probable hydroxyacid-oxoacid transhydrogenase & $4.6 \uparrow$ & $1.9 \times 10^{9}$ & 21 & 75.1 & 39.2 & 80 \\
\hline & NP_001014994.1 & Glycerol-3-phosphate dehydrogenase & $4.1 \uparrow$ & $3 \times 10^{9}$ & 30 & 90.6 & 40.1 & 248 \\
\hline & XP_001121241.2 & Probable serine/threonine-protein kinase clka & $4.0 \uparrow$ & $3.5 \times 10^{9}$ & 20 & 67.2 & 46.4 & 123 \\
\hline & XP_623995.1 & Endochitinase isoform 2 & $4.0 \uparrow$ & $5.3 \times 10^{9}$ & 35 & 63.1 & 61.2 & 312 \\
\hline & NP_001011563.1 & Juvenile hormone esterase precursor & $3.9 \uparrow$ & $2.5 \times 10^{10}$ & 41 & 73.7 & 63.9 & 300 \\
\hline & XP_393545.2 & Probable citrate synthase 1 & $3.7 \uparrow$ & $6.7 \times 10^{9}$ & 27 & 64.3 & 51.9 & 266 \\
\hline & XP_624408.2 & Trans-1,2-dihydrobenzene-1,2-diol dehydrogenase & $3.6 \uparrow$ & $2.8 \times 10^{10}$ & 35 & 86.4 & 43.1 & 447 \\
\hline & XP_006559841.1 & C-1-tetrahydrofolate synthase, cytoplasmic & $3.5 \uparrow$ & $6.7 \times 10^{9}$ & 60 & 71.7 & 101.5 & 349 \\
\hline & XP_006571053.1 & Peroxisomal multifunctional enzyme type 2 & $3.5 \uparrow$ & $9.5 \times 10^{9}$ & 46 & 65.7 & 80.5 & 348 \\
\hline & XP_623084.1 & Aldehyde dehydrogenase & $3.4 \uparrow$ & $2.3 \times 10^{10}$ & 45 & 92.2 & 55.7 & 436 \\
\hline & XP_393806.2 & Trifunctional enzyme subunit alpha, mitochondrial & $3.4 \uparrow$ & $4.4 \times 10^{9}$ & 41 & 61.4 & 84.3 & 245 \\
\hline & XP_393135.2 & $60 \mathrm{~S}$ ribosomal protein $123 \mathrm{a}$ & $3.3 \uparrow$ & $3.4 \times 10^{9}$ & 20 & 46.5 & 26.6 & 129 \\
\hline & XP_006558384.1 & Myosin regulatory light chain 2 & $16.6 \downarrow$ & $8.9 \times 10^{9}$ & 22 & 80.8 & 23.5 & 110 \\
\hline & XP_006560146.1 & Tropomyosin-1 & $9.6 \downarrow$ & $6.4 \times 10^{9}$ & 36 & 83.1 & 32.2 & 181 \\
\hline & XP_001120797.3 & Endocuticle structural glycoprotein sgabd-8 & $6.5 \downarrow$ & $6.3 \times 10^{9}$ & 3 & 20.8 & 13.8 & 37 \\
\hline & XP_006571119.1 & Calponin homology domain-containing protein & $5.7 \downarrow$ & $2.7 \times 10^{10}$ & 47 & 96.5 & 32.9 & 357 \\
\hline & XP_006559219.1 & Plasminogen activator inhibitor 1 RNA-binding protein & $5.1 \downarrow$ & $3.5 \times 10^{9}$ & 21 & 58 & 46.9 & 99 \\
\hline & XP_006558909.1 & Myosin light chain alkali & $4.5 \downarrow$ & $3.2 \times 10^{10}$ & 22 & 92.2 & 17.3 & 234 \\
\hline & XP_393381.2 & Peptidyl-prolyl cis-trans isomerase-like & $4.4 \downarrow$ & $5.9 \times 10^{10}$ & 30 & 76.1 & 22.8 & 296 \\
\hline & XP_006563462.1 & Uncharacterized abhydrolase domain-containing protein & $4.2 \downarrow$ & $5.8 \times 10^{9}$ & 67 & 56 & 115.5 & 282 \\
\hline & XP_001119962.2 & Titin & $3.7 \downarrow$ & $1 \times 10^{10}$ & 71 & 43 & 221.5 & 362 \\
\hline & XP_001121746.1 & Neurofilament heavy polypeptide & $3.7 \downarrow$ & $4.1 \times 10^{11}$ & 36 & 66.2 & 52.0 & 929 \\
\hline & NP_001035314.1 & Odorant binding protein 13 precursor & $3.3 \downarrow$ & $1.5 \times 10^{11}$ & 28 & 81.1 & 15.1 & 551 \\
\hline & XP_006563164.1 & Pterin-4-alpha-carbinolamine dehydratase & $3.2 \downarrow$ & $3.7 \times 10^{9}$ & 9 & 60.1 & 16.9 & 60 \\
\hline & XP_006565471.1 & Large proline-rich protein BAG6 & $3.2 \downarrow$ & $1.3 \times 10^{9}$ & 33 & 49.6 & 131.0 & 145 \\
\hline & XP_392826.1 & Small ubiquitin-related modifier-like & $3.0 \downarrow$ & $3.3 \times 10^{9}$ & 10 & 72.4 & 11.2 & 80 \\
\hline & XP_006559096.1 & ATPase inhibitor mai-2, mitochondrial & $2.9 \downarrow$ & $1.1 \times 10^{10}$ & 8 & 59.2 & 11.7 & 107 \\
\hline \multirow[t]{30}{*}{ Drone } & XP_006558384.1 & Myosin regulatory light chain 2 & $12.1 \uparrow$ & $8.9 \times 10^{9}$ & 22 & 80.8 & 23.5 & 110 \\
\hline & XP_006558182.1 & Tropomyosin-2 & $7.2 \uparrow$ & $1.7 \times 10^{10}$ & 49 & 87.7 & 32.9 & 279 \\
\hline & XP_006567675.1 & Uncharacterized protein LOC725148 & $4.6 \uparrow$ & $8.2 \times 10^{9}$ & 25 & 88.1 & 28.5 & 158 \\
\hline & XP_006568746.1 & Troponin I & $4.4 \uparrow$ & $6.5 \times 10^{9}$ & 26 & 64.6 & 24.8 & 128 \\
\hline & XP_006558909.1 & Myosin light chain alkali & $4.2 \uparrow$ & $3.2 \times 10^{10}$ & 22 & 92.2 & 17.3 & 234 \\
\hline & XP_006572093.1 & Transcription elongation regulator 1 & $4.1 \uparrow$ & $4.2 \times 10^{10}$ & 25 & 74.7 & 37.0 & 381 \\
\hline & XP_006557549.1 & Troponin $\mathrm{T}$, skeletal muscle & $3.9 \uparrow$ & $1.6 \times 10^{10}$ & 42 & 55.5 & 47.0 & 296 \\
\hline & XP_006564366.1 & Titin & $3.8 \uparrow$ & $7.1 \times 10^{9}$ & 185 & 13.4 & 2239.4 & 444 \\
\hline & XP_006567699.1 & Neurochondrin homolog & $3.7 \uparrow$ & $2.7 \times 10^{9}$ & 32 & 48.3 & 84.2 & 132 \\
\hline & XP_006566313.1 & Troponin C type I & $3.7 \uparrow$ & $6.8 \times 10^{9}$ & 18 & 71.7 & 17.4 & 161 \\
\hline & XP_006562528.1 & LON protease homolog, mitochondrial-like isoform X1 & $3.4 \uparrow$ & $1.7 \times 10^{9}$ & 39 & 51.5 & 112.9 & 127 \\
\hline & XP_393281.2 & Paramyosin, long form & $3.2 \uparrow$ & $6.6 \times 10^{10}$ & 131 & 86.4 & 102.1 & 1054 \\
\hline & XP_006569863.1 & Myosin heavy chain, muscle & $3.1 \uparrow$ & $3.5 \times 10^{11}$ & 319 & 80.5 & 224.9 & 3138 \\
\hline & XP_006557649.1 & Hexokinase type 2 & $2.9 \uparrow$ & $8 \times 10^{8}$ & 28 & 69.5 & 50.0 & 91 \\
\hline & XP_006568334.1 & Pupal cuticle protein & $2.8 \uparrow$ & $8.2 \times 10^{9}$ & 11 & 48.7 & 31.1 & 157 \\
\hline & XP_003251110.1 & Uncharacterized protein LOC100578085 & $2.1 \downarrow$ & $4.5 \times 10^{9}$ & 25 & 74.7 & 32.3 & 146 \\
\hline & XP_393452.3 & Pro-resilin-like & $2.0 \downarrow$ & $6.1 \times 10^{9}$ & 3 & 52.5 & 17.4 & 57 \\
\hline & NP_001136109.1 & Glycine-rich cuticle protein & $1.9 \downarrow$ & $5.2 \times 10^{9}$ & 7 & 27.1 & 30.7 & 99 \\
\hline & XP_006567467.1 & Fatty acid synthase & $1.9 \downarrow$ & $9.1 \times 10^{9}$ & 92 & 55.3 & 264.4 & 275 \\
\hline & XP_001121640.3 & Pupal cuticle protein & $1.7 \downarrow$ & $1 \times 10^{9}$ & 6 & 30.4 & 36.2 & 48 \\
\hline & XP_001121782.1 & Proline-rich protein 4 & $1.6 \downarrow$ & $2 \times 10^{9}$ & 9 & 63.4 & 16.6 & 48 \\
\hline & XP_006561116.1 & Fibrillin-2 & $1.6 \downarrow$ & $8.7 \times 10^{8}$ & 16 & 9.1 & 283.9 & 66 \\
\hline & XP_003249362.1 & Uncharacterized protein LOC100577680 & $1.4 \downarrow$ & $8.9 \times 10^{10}$ & 36 & 81.2 & 41.3 & 633 \\
\hline & XP_006569431.1 & Heterogeneous nuclear ribonucleoprotein $\mathrm{R}$ & $1.4 \downarrow$ & $3.2 \times 10^{8}$ & 59 & 83.6 & 64.1 & 20 \\
\hline & XP_001120418.1 & Cuticle protein 18.7 & $1.3 \downarrow$ & $5.9 \times 10^{10}$ & 13 & 75.1 & 17.6 & 249 \\
\hline & NP_001035323.1 & Cytochrome P450 4G11 & $1.2 \downarrow$ & $7.8 \times 10^{8}$ & 19 & 43.1 & 62.3 & 75 \\
\hline & XP_003250694.1 & Uncharacterized protein LOC100577362 & $1.2 \downarrow$ & $1.7 \times 10^{9}$ & 5 & 36.8 & 20.0 & 61 \\
\hline & XP_006560156.1 & Uncharacterized protein LOC102653832 & $1.2 \downarrow$ & $4.2 \times 10^{10}$ & 19 & 60.6 & 41.6 & 360 \\
\hline & XP_003250245.1 & Uncharacterized protein LOC100576126 & $1.2 \downarrow$ & $4.8 \times 10^{9}$ & 29 & 54.8 & 56.9 & 186 \\
\hline & NP_001011572.1 & Transferrin 1 precursor & $1.1 \downarrow$ & $1.2 \times 10^{11}$ & 107 & 94.7 & 78.7 & 1220 \\
\hline
\end{tabular}

RFC), calponin (XP_006571119.1; 5.7 RFC) and myosin light chain (XP_006558909.1; 4.5 RFC).

The most differentially abundant proteins in parasitized drones in comparison to the unparasitized controls were myosin regulatory light chain 2 (XP_006558384.1) with a RFC of 12.1, tropomyosin-2 (XP_006558182.1; 7.2 RFC), an uncharacterized protein LOC725148 (XP_006567675.1; 4.6 RFC), troponin I (XP_006568746.1; 4.4 RFC), myosin light chain protein (XP_006558909.1; 4.2 RFC) and transcription elongation regulator 1 (XP_006572093.1; 4.1 RFC). Proteins of highest abundance in non-parasitized drones in comparison to their parasitized counterparts included an uncharacterized protein LOC100578085 (XP_003251110.1; 2.1 RFC), pro-resilin (XP_393452.3; 2.0 RFC), a glycine-rich cuticle protein (NP_001136109.1, 1.9 RFC) and a pupal cuticle protein (XP_001121640.3, 1.7 RFC).

Of the combined set of 52 SSDA proteins identified in both castes in response to parasitization by Varroa, 21 showed a similar expression 
profiles (i.e. the same direction in fold change in both castes in parasitized and non-parasitized states). However significant differences were observed between both castes in response to parasitization particularly in proteins associated with metabolism and muscle structure and function (Table 2). The proteins with the greatest divergence in abundance were myosin regulatory light chain 2 (XP_006558384.1), tropomyosin-1 (XP_006560146.1), myosin light chain (XP_006558909.1), calponin (XP_006571119.1) and actin (XP_623619.1) which were all of higher abundance in parasitized drones and lower abundance in parasitized workers in comparison to their respective controls. To ensure that these specific differences in abundances of these proteins are associated with response to Varroa and not due to the inherent difference between castes a $t$-test was conducted between the unparasitized proteomes of worker and drone. In total the abundance of 393 proteins were statistically significantly $(\mathrm{p}<0.05$; Supplementary Table 1e; Table 2) altered and the muscle associated proteins of higher abundance in parasitized drones are found in higher endogenous abundances in workers which indicates that these proteins are expressed at higher abundances specifically in drones in response to parasitization.

An alteration to the abundance of cuticle component proteins can be seen in both worker and drone pupae when parasitized by Varroa (Supplementary Tables $1 \mathrm{c}$ and d respectively). Endocuticle-structural glycoprotein is present at 6.5 times lower RFC in parasitized worker pupae, with cuticular protein 2 precursor and cuticular protein 12 precursor present at $-1.41 \mathrm{RFC}$ and $2 \mathrm{RFC}$ respectively, compared to levels in control workers. Pupal cuticle protein (2.8 RFC), endocuticle structural protein (1.86 RFC), cuticular protein 27 precursor (1.86, $\mathrm{RFC})$, cuticular protein 2 precursor $(-1.62 \mathrm{RFC})$, cuticular protein 16 precursor $(-1.86 \mathrm{RFC})$, cuticular protein 1 precursor $(-1.86 \mathrm{RFC})$, glycine-rich cuticle protein $(-1.9 \mathrm{RFC})$, cuticular protein CPF1 precursor ( $-2 \mathrm{RFC}$ ), cuticular protein GIA ( $-2 \mathrm{RFC}$ ), cuticle protein 18.7 $(-1.3 \mathrm{RFC})$ and an additional pupal cuticle protein $(-1.7)$ were all present at altered levels of detectable abundance in the parasitized drone pupae compared to control drone pupae.

A number of immune proteins and those with proposed functions in the healing/ clotting response were altered in abundance in the parasitized drone and worker pupae, in a non-correlating manner. Parasitized worker pupae showed reduced abundance of apolipoprotein III ( -1.74 RFC) compared to control. Toll -like receptor 13-like was present at 1.62 higher RFC in parasitized workers. Protein croquemort was not detected in the unparasitized worker pupae, and was deemed exclusively expressed in the parasitized pupae (Supplementary Table 1b (i)) A number of proteins from the serine superfamily were altered in abundance in both worker and drone sample groups. The abundance of Serine/threonine kinase clKA (4 RFC), serine-tRNA ligase (2.29 RFC) and serine hydroxymethyltransferase (3.4 RFC) (Supplementary Table 1c) was higher in parasitized worker pupae, with serine/arginine repetitive matrix protein 2-like (1.62 RFC), transmembrane protease serine 9 (1.32 RFC), antitrypsin-like $(-1.23 \mathrm{RFC})$, serine hydroxymethyltransferase $(-1.23 \mathrm{RFC})$ serine protease ester $(-1.41 \mathrm{RFC})$ altered in the parasitized drone pupae (Supplementary Table 1d). Comparison of parasitized drone pupae to their control identified MD-2 lipid recognition protein which was absent at a detectable level in unparasitized control drone pupae (Supplementary Table 1b(ii)). Serine protease ester was reduced in parasitized drones $(-1.5 \mathrm{RFC})$, and apolipoprotein III precursor was detected at -2 RFC (Supplementary Table 1d). Transferrin precursor was present at 2.14-fold lower in the parasitized drone pupae, as was fibrillin-2, detectable at levels of 3-fold lower when drone pupae were parasitized. Apolipoprotein D-like was present at a RFC of 1.41 higher in parasitized drones compared to control.

The intensity values for proteins that were absent from a given sample were replaced through a data imputation step which permitted statistical and functional analyses to be conducted on all identified proteins (including exclusively detected proteins). Hierarchical clustering of all statistically significant and exclusively expressed proteins (Fig. 1B) resolved 15 distinct groups of proteins based on similarities in protein abundances and expression profile (Supplementary Table 1e). Group 5 highlights proteins with similar profiles in response to parasitization in both drones and workers and include cuticular proteins which were of lower abundance in both drone and worker when parasitized and also a number of ribonucleotide binding proteins which were of higher abundances in both castes. A number of groups highlight a divergent response between castes in response to Varroa parasitization including group 13 which comprises proteins that of low abundance in non-parasitized workers and group 15 comprising proteins that are of low abundance in parasitized and non-parasitized drones, low abundance in non-parasitized workers but high abundances in parasitized workers. This latter group comprised proteins associated with metabolism and the ribosome. Volcano plots (Fig. 2) were also generated for both parasitized and non-parasitized drone and worker proteins to display individual proteins most differentially abundant under parasitization.

To further characterize the pathways and processes involved in response to Varroa a STRING and KEGG analysis was conducted on proteins of higher and lower abundances in each caste. No significant clusters were identified in the proteins that were of lower abundance in parasitized drones and workers. A number of clusters were identified in the proteins of higher abundance in parasitized drones including proteins associated with Oxidative phosphorylation, the TCA cycle, fatty acid metabolism and amino acid metabolism (Fig. 3A). Although the same processes were present in the proteins of higher abundance in parasitized workers a greater number of individual proteins associated with these processes were observed. In addition, proteins involved in translation and protein processing in endoplasmic reticulum were exclusively observed to be of higher abundance in workers (Fig. 3B). KEGG pathway analysis also highlights a higher prevalence of metabolism proteins in parasitized workers in comparison to parasitized drones (Supplementary Fig. 1A and B).

\section{Discussion}

Varroa infestation of $A$. mellifera colonies is widely regarded as one of the main causes of colony weakening and loss, particularly at high levels of infestation (Evans et al., 2009, Le Conte et al., 2010, Genersch, 2010). Varroa have long been speculated to weaken the bee and thus the colony as a whole, due to the suppression of their immune response which may leave them susceptible to a variety of pathogens. As Varroa demonstrate no exclusivity towards drone larvae in colonies of $A$. mellifera, both worker and drone pupae were chosen for investigation. The aim of this study was to characterize the effect of parasitization by Varroa on A. mellifera drone and worker pupae at the molecular level and to investigate whether Varroa had different effects on worker and drone pupae.

Based on this quantitative proteomic analysis there is a clear difference not only between the proteomes of drone and worker pupae but also in the extent of response in workers and drones to parasitization by Varroa (Fig. 1A). Hierarchical clustering of statistically significant differentially abundant and exclusively expressed proteins resolved a number of proteins with similar expression profiles (Fig. 1B). Some of these groupings highlighted a conserved response to Varroa including differential abundances in proteins associated with cuticle, metabolism and innate immunity. However, a number of significant caste specific responses were also observed, with muscle and structural proteins displaying a strong disparity in expression levels (Table 2). Proteins involved in fatty acid and carbohydrate metabolism were more abundant in parasitized workers as were a large number of ribosomal proteins. These proteins groups, expression profiles with respect to both castes and their significance are discussed in detail below. 


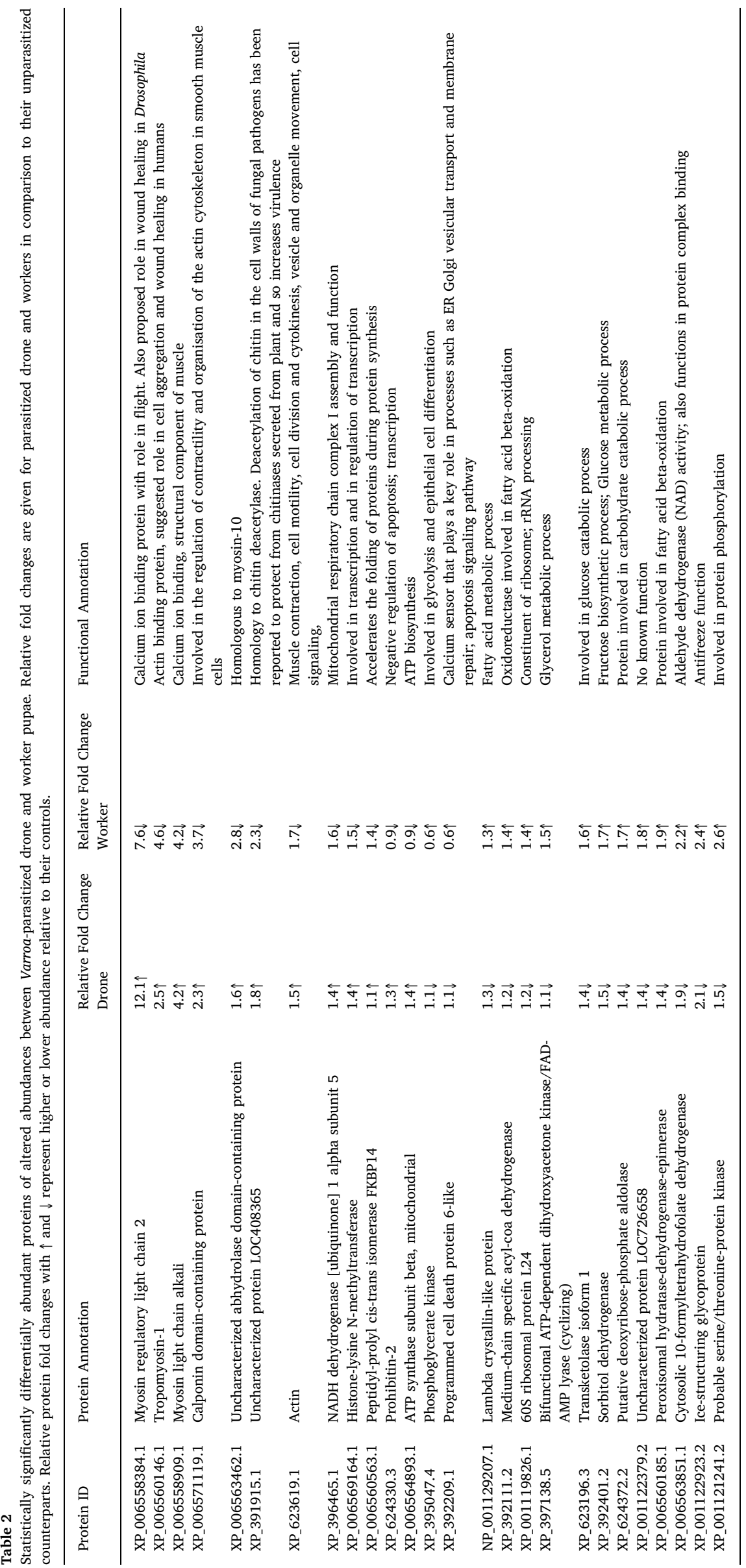


A Drone Pupae

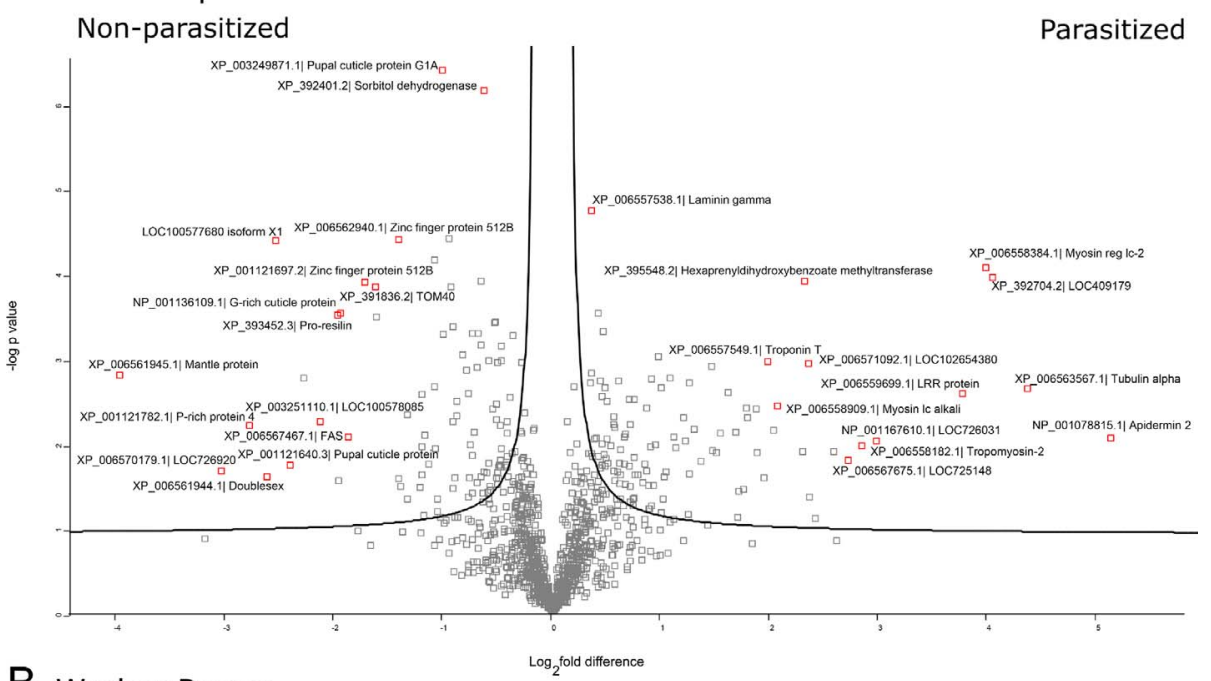

B Worker Pupae Non-parasitized

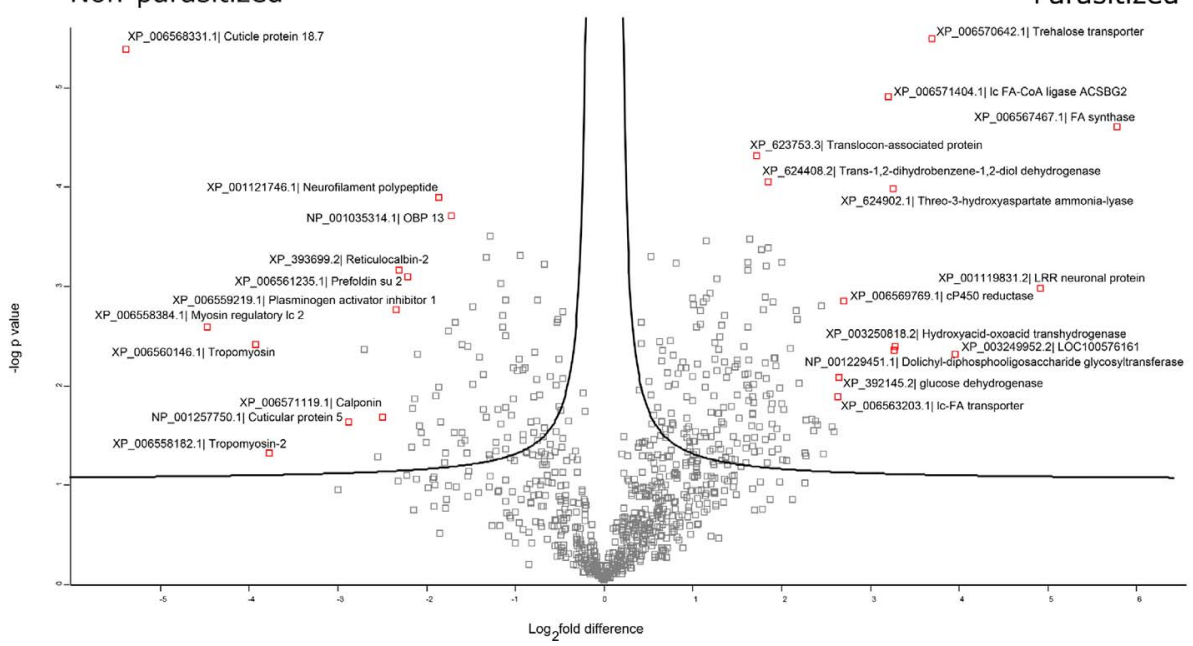

Fig. 2. Volcano plots highlighting differentially expressed proteins in parasitized and non-parasitized drone and worker pupae. Volcano plots showing the distribution of quantified proteins according to $\mathrm{p}$ value $\left(-\log _{10} \mathrm{p}\right.$-value) and fold change ( $\log _{2}$ mean intensity difference). Proteins above the curved line are statistically significant and those to the right and left of the vertical lines indicate fold changes greater than 1.2 in (A) parasitized and unparasitized drone pupae respectively and (B) parasitized and unparasitized worker pupae respectively.

\subsection{Metabolic proteins}

Examination of the total proteomic profiles of parasitized worker and drone pupae highlights alterations to the abundance of a significant number of proteins involved in cell metabolism. Through STRING analysis, the most enriched biological pathways in parasitized worker and drone pupae are metabolic pathways (Fig. 3). During feeding, the Varroa female and progeny ingest substantial amounts of haemolymph, and must compete with the growing pupa for nutrients (De Jong et al., 1982, Le Conte et al., 2010). The upregulation of metabolic associated proteins and those involved in energy production could be due to an effort by the host to replenish lost metabolites, or could be due to manipulation by the parasite to ensure successful feeding. Fatty acid synthase was exclusively detected in the parasitized worker pupae, and absent from controls. This protein is predominantly involved in fatty acid synthesis, but also involved in cell differentiation and growth (Arrese and Soulages, 2010, Stanley-Samuelson et al., 1988). Another protein involved in fatty acid synthesis and metabolism with an additional role in cell differentiation was exclusively detected in the parasitized worker pupae, was long chain fatty acid CoA ligase (Tomoda et al., 1991). The extra abundance of these proteins could indicate a manipulation by the mite to benefit from a richer haemolymph. Hexokinase, present at an increased level of abundance in the parasitized drone pupae, is a positive regulator of cell proliferation and growth, and its elevated presence in the parasitized drone could support extra growth (Gwak et al., 2005, Robey and Hay, 2006). Changes to the growth patterns of the host, extra production of fatty acids or sugars in haemolymph or boosted carbohydrate metabolism could benefit the parasite each time it feeds (Vinson and Iwantsch, 1980). Tobacco hornworm larvae parasitized by Cotesia congregata show increased growth and metabolic efficiency. The study also highlighted a correlation between growth increase and level of parasitization (Alleyne and Beckage, 1997). There was a significant increase in the relative abundance of a trehalose transporter in the parasitized worker pupae, which was absent from detection in the control worker pupae. Trehalose is the main blood sugar in insect haemolymph, and its increase could enrich the blood quality for the parasitizing mite (Liu et al., 2013). Trehalose is regarded as an important nutrient in Anopheles gambiae during infection by Plasmodium (Liu et al., 2013). The concentration of trehalose within the haemolymph of Manduca sexta parasitized by Cotesia congregata was significantly higher than in larvae than were unparasitized (Thompson et al., 2005). A similar response has also been found in larvae of Trichoplusia ni which showed higher concentrations of protein, trehalose and glycogen compared with larvae that were unparasitized by Hyposoter exiguae (Thompson, 1982). Enhanced metabolism and protein biosynthesis in A. mellifera pupal haemolymph has been associated with increased immunity to Varroa (Hu et al., 2016). Although on emergence parasitized worker and drone A. mellifera are lighter that their 
A

Up in Parasitized Drone Pupae

\begin{tabular}{lcc}
\hline Pathway description & $\begin{array}{c}\text { Count in } \\
\text { gene set }\end{array}$ & FDR \\
\hline Metabolic pathways & 31 & $2.85 \mathrm{E}-12$ \\
Oxidative phosphorylation & 12 & $1.55 \mathrm{E}-09$ \\
Citrate cycle (TCA cycle) & 6 & $7.54 \mathrm{E}-06$ \\
Fatty acid metabolism & 6 & $9.75 \mathrm{E}-06$ \\
$\begin{array}{l}\text { Valine, leucine and isoleucine } \\
\text { degradation }\end{array}$ & 5 & 0.000113 \\
Propanoate metabolism & 4 & 0.000275 \\
\hline
\end{tabular}

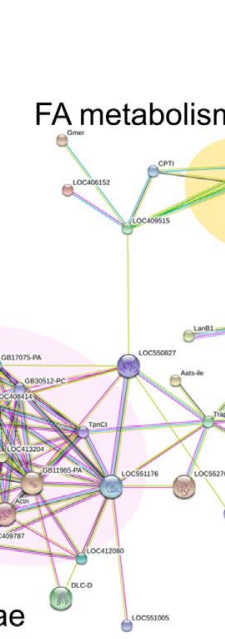

B
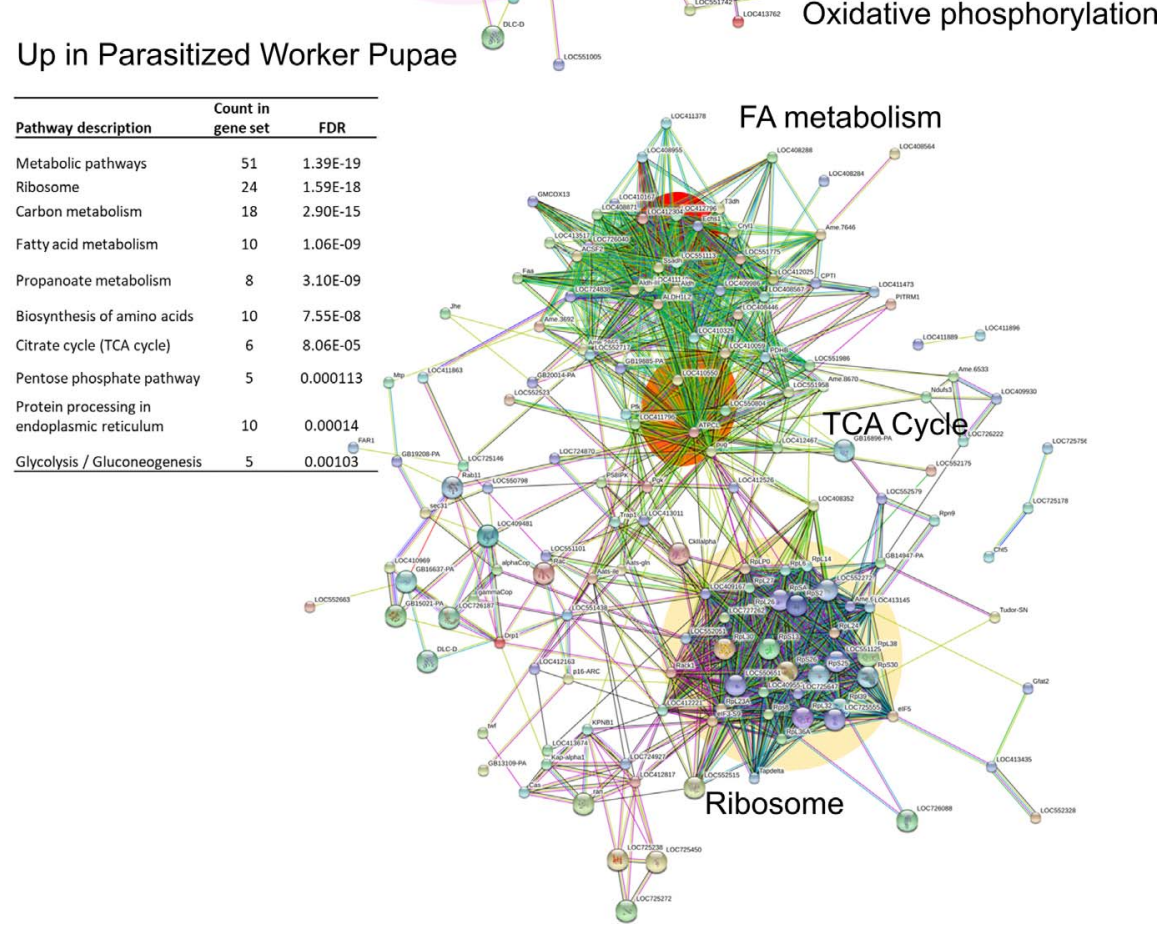

Fig. 3. Interaction network analysis of up regulated proteins in parasitized pupae Protein interaction information was obtained from the STRING database using gene lists extracted for statistically significant differentially abundant (SSDA) proteins of increased abundance in (A) parasitized drones and (B) parasitized workers. Statistically enriched KEGG and Gene Ontology (GO) terms were examined to identify pathways and processes enriched within each set of proteins. unparasitized counterparts, perhaps the weight loss would be much greater if manipulation of growth response pathways had not taken place (Bowen-Walker and Gunn, 2001).

\subsection{Cuticle component proteins}

Varroa feed communally and repeatedly though a shared opening in the cuticle that exhibits a delayed healing response (Kanbar and Engels, 2004). On examining the proteomic expression profiles of both worker and drone pupae, there was a reduction in the relative abundance of a significant number of cuticle component proteins. Pupal cuticle proteinlike was present at a reduced level of over $3 \mathrm{RFC}$ in the parasitized drone pupae, as was cuticle protein 18.7-like cuticle protein, present at reduced levels of almost 2.5 RFC. In parasitized worker pupae, endocuticle structural glycoprotein SgAbd-8 was reduced at almost 6.5 RFC compared to worker control. Apidermin 3 precursor was also reduced in the parasitized worker pupae at a level of over 2 RFC, with cuticular protein 12 precursor present at a reduced level upon parasitization, over 2 -fold. The reduced abundance of these cuticle associated proteins following parasitization by Varroa could indicate a compromised healing response of the cuticle in order to allow the
Varroa to feed without the need to open a new aperture in the cuticle each time (Richards et al., 2011).

\subsection{Muscle proteins}

One of the most striking differences between the response of drone and worker pupae to Varroa parasitization is in the relative abundance of muscle associated proteins (Table 2). Proteins such as myosin regulatory light chain 2 , tropomyosin-1, myosin light chain alkali and actin were increased in abundance in parasitized drones but reduced in worker pupae. Although the physiology of drone and worker pupae differ in many respects the reason for the difference in abundance of muscle proteins is unclear but it is possible that parasitism by Varroa of drone pupae may stimulate growth while worker pupae respond by showing reduced growth. Drones are larger on emergence (270-290 mg) than workers (144-162 mg) and contain more protein (65-97 $\mathrm{mg}$ vs $25-37 \mathrm{mg}$ ) therefore may be better able to withstand the effects of parasitisation. Interestingly, hexokinase (Table 1) is present at an increased level of abundance in parasitized drone pupae, but not worker pupae, and is a positive regulator of growth (Gwak et al., 2005; Robey and Hay, 2006). Transcription elongation regulator 1 was 
exclusively expressed in parasitized drones and also supports enhanced growth.

\subsection{Anticoagulant proteins and the healing response}

While the humoral component of the blood clotting response of insects and mammals is dissimilar, they share many similarities in the cellular components of the clotting cascade. This consists of the release of granular components and the formation of micro particles from the formation of membranous vesicles released from cells in the blood or haemolymph (Theopold et al., 2002). The formation of these particles is calcium dependent and calcium has also been linked with platelet binding with high levels leading to increased aggregation (Strukova, 2006). Following injury to the insect cuticle, defenses are orchestrated by the fat body, the hemocoel and the hemocytes. Wound healing occurs through clotting factors hemolectin and Eig71Ee, crosslinking of which occurs in a calcium dependent manner (Parker et al., 2012). A significant number of proteins with proposed roles in calcium binding were present at much lower levels of abundance in the parasitized worker pupae. Calmodulin-like protein was present at over 2-fold reduced abundance in the parasitized worker pupae. Calmodulin has shown to be present at higher levels in the cells immediately surrounding wounds (Narciso et al., 2015). Calponin homology domaincontaining protein DDB_G0272472-like was also present at a reduced level in the parasitized worker pupae, at levels of over 5.6 RFC. These proteins are predominantly involved in calcium binding, and are also over-expressed at wound sites in human epidermal cells, suggesting a possible role in wound healing (Lansdown, 2002). A recent study using Drosophila provided further evidence that calcium signaling is implicated in the wound healing response following injury to imaginal disks and observation of calcium communication (Restrepo and Basler, 2016). Calumenin, an additional calcium binding protein was also reduced in abundance upon parasitization in the worker pupae at levels of almost 2.5 RFC (Vorum et al., 2000). Reticulocalbin-2 was present at much lower levels in the parasitized worker pupae. The exact function of this protein has not been elucidated, but it is thought to participate in calcium binding (Ludvigsen et al., 2009). The alteration in the abundance of proteins involved in calcium signaling was not evident in the parasitized drone pupae. One protein implicated in the clotting response in drone pupae was antithrombin-III, present 1.7-fold lower in parasitized samples. This protein is similar to human anti-thrombin, and is an important regulator of coagulation which down regulates fibrinolysis in blood (Kanost, 2013). Deficiencies of this protein in humans leads to an excessive bleeding disorder, and its significant reduction in the bees that were parasitized by Varroa in this instance could indicate an anti-coagulant effect induced by the mite to facilitate uninterrupted feeding (Fay et al., 1997, Marcos-Contreras et al., 2013). Fibrillin-2 was also present at lower levels in the drone pupae that were parasitized; at over 3-fold difference. This protein is an extra cellular matrix protein, heavily involved in the healing response (Brinckmann et al., 2010)

\subsection{Proteins involved in the immune response}

There is considerable debate on the nature of the Varroa-Apis interaction and whether the mites possess the ability to suppress the host's immune response (Gregory et al., 2005, Kuster et al., 2014, Khongphinitbunjong et al., 2015, Koleoglu et al., 2017). Through label free analysis of the parasitized worker and drone pupae, the proteins significantly altered in abundance were examined for the presence of immune factors. The presence of a clear alteration in the immune response of either drone or worker pupae is not evident. Apolipoprotein III was present at reduced levels in both parasitized drone and worker at levels of 1.86 and 1.74 RFC respectively. Apolipoprotein D-like and apolipophorins were both present at reduced levels of 1.4 RFC I drone pupae that were parasitized by Varroa. The apolioproteins have been linked with a central role in the immune response, including LPS detoxification, phagocytosis and also pattern recognition (Whitten et al., 2004). Protein croquemort was only present in the parasitized worker pupae, and absent at a detectable level in control workers. This protein is involved in phagocytosis (Franc et al., 1999) and the increased abundance could be indicative of an activated immune response to the presence of Varroa. In the parasitized worker and drone pupae proteomes there is evidence of alterations to a substantial number of serine proteases, such a serine protease $\mathrm{K} 12 \mathrm{H} 4.7$, which is heavily involved in the immune response (Van Vaerenbergh et al., 2015). There was also an increased abundance following parasitization of serine/threonine kinase cLKA, by 4 -fold, which could further indicate an elevated immune response in the worker pupae that were parasitized during development to purple eye stage (Bania et al., 1999, China et al., 2016). This protein however, was detected at lower abundance levels in the parasitized drone pupae by over 1.5 RFC. MD-2 related lipid recognition protein was only detected in the parasitized drone pupa, ad absent form control drones. This protein is involved in the Toll-like signaling pathway which is an important feature of the humoral immune response (Inohara and Nuñez, 2002, Gay and Gangloff, 2007). Its presence in the parasitized drone pupae may indicate activation of an immune response due to the presence of the feeding Varroa. Examination of the alterations to the abundance of immune related proteins is suggestive of a modest effect of Varroa on the immune response, and on examining the abundance of certain immune proteins, highlights an activated immune response to Varroa parasitization. The use of whole body proteomics was employed to give an overall view of the effect of parasitization on the whole proteome but it is also worth noting that analysis of fat body of haemolymph protein separately could offer further clarification on the effect of Varroa on the immune system.

\subsection{Ribosomal proteins}

A large group of ribosomal component associated proteins were altered in abundance in the comparison between unparasitized and parasitized worker pupae, highlighted using STRING analysis (Fig. 3A) The majority of the ribosomal proteins were present at increased abundance in the parasitized workers, with just two out of the sixteen significantly varied proteins exhibiting a drop in their relative abundance. Previous studies have shown that ribosomal proteins may regulate and modify cell growth patterns independently of their previously well-established role as components of an organism's translational apparatus (Naora and Naora, 1999) and an increased abundance of ribosomal proteins was found in Varroa sensitive hygiene bees (Hu et al., 2016). Mutations of the ribosomal gene s6 in Drosophila lead to increased tissue growth, supporting the idea that ribosomal genes can alter cell differentiation and growth (Stewart and Denell, 1993, Montagne et al., 1999).

\subsection{Neurological development proteins}

Weakened A. mellifera colonies are characterized by reduced numbers of workers with insufficient numbers to continue the life of the hive (Williams et al., 2010, Evans et al., 2009). One possibility is that worker bees are unable to return to the hive after foraging due to cognitive impairment (Henry et al., 2012). Cognitive impairment caused by Varroa may be direct through lowered levels of nutrition during development or indirect through the heightening of other life systems within the bee due to the stress of parasitization, causing impaired neuron development (Zera and Harshman, 2001). An analysis of the abundance of memory associated proteins revealed that a number of proteins linked to memory were present at lower abundance upon parasitization. Uncharacterized protein XP_003249362 identified as homologous to Protein Appl-like using the bLINK resource, has been shown as essential for memory in Drosophila, and was present at an abundance of -2.62 RFC in parasitized drones. Protein lingerer-like, an 
additional protein linked to long term memory formation in Drosophila, was reduced in abundance in the parasitized worker pupae. In Varroa parasitized worker and drone pupae, there were two odorant binding proteins reduced in abundance in each group. These proteins are thought to play a role in memory formation through olfactory sensing, and the reduction here, particularly in the parasitized worker pupae, could have a negative effect on ability to forage or return to the colony following foraging (Forêt and Maleszka, 2006, Davis, 2005). Adult bees showing Varroa sensitive hygiene display increased abundance of odorant binding protein and this may enable the bee to respond to Varroa infection and trigger a specific response to remove the mite (Hu et al., 2016). Adult worker bees that are parasitized by Varroa appear to be morphologically normal, but exhibit a decreased learning capacity (Navajas et al., 2008). Workers also exhibit reduced capability for foraging, including prolonged absences from the hive with a lower rate of return. Prefoldin subunit 2-like was absent from detection in the parasitized worker pupae and in Drosophila depletion of this protein resulted in overgrowth of neuroblasts in the brain of the fly, which could lead to incorrect functioning (Zhang et al., 2016).

\section{Conclusion}

In this study a complete comparative analysis of the effect of parasitization by $V$. destructor on the proteomes of drone and worker honeybee pupae is presented. A higher level of proteomic change was evident in the parasitized worker pupae with almost double the number of SSDAs as evident in the parasitized drone pupae. The effect of Varroa on the immune response of both drone and worker A. mellifera appears to be modest. There is a clear reduction to the healing ability of the bee evident through a substantial reduction in the abundance of various cuticular proteins, and the loss in the levels of proteins involved in coagulation of hemolymph. These findings support results from other studies into the mechanisms by which blood/ hemolymph feeding parasites manipulate the host to alter the flow and availability of blood. A reduction in the abundance of proteins involved in nervous system development, and in olfactory sensory perception was also demonstrated and this could be detrimental to the colony if infestation levels are high and a large proportion of the foraging workers are parasitized.

\section{Acknowledgements}

C. Surlis was the recipient of a government of Ireland postgraduate scholarship from the Irish Research Council. This work was part funded by a grant from the Federation of Irish Beekeepers Associations. QExactive mass spectrometer was funded under the SFI Research Infrastructure Call 2012; Grant Number: 12/RI/2346 (3) to Prof S. Doyle.

\section{Appendix A. Supplementary data}

Supplementary data associated with this article can be found, in the online version, at http://dx.doi.org/10.1016/j.jinsphys.2017.12.004.

\section{References}

Alleyne, M., Beckage, N.E., 1997. Parasitism-induced effects on host growth and metabolic efficiency in tobacco hornworm larvae parasitized by Cotesia congregata. J. Insect Physiol. 43, 407-424.

Arrese, E.L., Soulages, J.L., 2010. Insect fat body: energy, metabolism, and regulation. Annu. Rev. Entomol. 55, 207.

Bania, J., Stachowiak, D., Polanowski, A., 1999. Primary structure and properties of the cathepsin G/chymotrypsin inhibitor from the larval hemolymph of Apis mellifera. Eur. J. Biochem. 262, 680-687.

Beaurepaire, A.L., Truong, T.A., Fajardo, A.C., Dinh, T.Q., Cervancia, C., Moritz, R.F., 2015. Host specificity in the honeybee parasitic mite, Varroa spp. in Apis mellifera and Apis cerana. PloS one 10, e0135103.

Bowen-Walker, P.L., Gunn, A., 2001. The effect of the ectoparasitic mite, Varroa destructor on adult worker honeybee (Apis mellifera) emergence weights, water, protein, carbohydrate, and lipid levels. Entomol. Exp. Appl. 101, 207-217.
Brinckmann, J., Hunzelmann, N., Kahle, B., Rohwedel, J., Kramer, J., Gibson, M.A., Hubmacher, D., Reinhardt, D.P., 2010. Enhanced fibrillin-2 expression is a general feature of wound healing and sclerosis: potential alteration of cell attachment and storage of TGF-[beta]. Lab. Invest. 90, 739.

Büchler, R., Drescher, W., Tornier, I., 1992. Grooming behaviour of Apis cerana, Apis mellifera and Apis dorsata and its effect on the parasitic mites Varroa jacobsoni andTropilaelaps clareae. Exp. Appl. Acarol. 16, 313-319.

Chen, C., Liu, Z., Luo, Y., Xu, Z., Wang, S., Zhang, X., Dai, R., Gao, J., Chen, X., Guo, H., 2017. Managed honeybee colony losses of the Eastern honeybee (Apis cerana) in China (2011-2014). Apidologie 48, 692-702.

China, C., Wang, Yu, Xiu, Jiang Fan, Cheng, Jin Zhi, Luo, Man, Zhao, Peng, Shang, Xiao Li, Wang, Tao, Wu, Jian Wei, 2016. Proteomic Analysis of the Peritrophic Matrix from the Midgut of Third Instar Larvae, Musca domestica. Biomed Environ Sci 29, $56-65$.

Davis, R.L., 2005. Olfactory memory formation in Drosophila: from molecular to systems neuroscience. Annu. Rev. Neurosci. 28, 275-302.

de Jong, D., de Jong, P., Goncalves, L., 1982. Weight loss and other damage to developing worker honeybees from infestation with Varroa jacobsoni. J. Apicultural Res. 21, 165-167.

Evans, J.D., Saegerman, C., Mullin, C., Haubruge, E., Nguyen, B.K., Frazier, M., Frazier, J., Cox-Foster, D., Chen, Y., Underwood, R., Tarpy, D.R., 2009. Colony collapse disorder: a descriptive study. PloS one 4 (8), e6481.

Fang, Y., Feng, M., Han, B., Lu, X., Ramadan, H., Li, J., 2014. In-depth proteomics characterization of embryogenesis of the honey bee worker (Apis mellifera ligustica) Mol. Cell. Proteomics 13, 2306-2320.

Fang, Y., Feng, M., Han, B., Qi, Y., Hu, H., Fan, P., Huo, X., Meng, L., Li, J., 2015. Proteome analysis unravels mechanism underling the embryogenesis of the honeybee drone and its divergence with the worker (Apis mellifera lingustica). J. Proteome Res. 14, 4059-4071.

Fay, W.P., Parker, A.C., Condrey, L.R., Shapiro, A.D., 1997. Human plasminogen activator inhibitor-1 (PAI-1) deficiency: characterization of a large kindred with a null mutation in the PAI-1 gene. Blood 90, 204-208.

Forêt, S., Maleszka, R., 2006. Function and evolution of a gene family encoding odorant binding-like proteins in a social insect, the honey bee (Apis mellifera). Genome Res. $16,1404-1413$.

Franc, N.C., Heitzler, P., White, K., 1999. Requirement for croquemort in phagocytosis of apoptotic cells in Drosophila. Science 284 (5422), 1991-1994.

Fries, I., Lindström, A., Korpela, S., 2006. Vertical transmission of American foulbrood (Paenibacillus larvae) in honey bees (Apis mellifera). Veterinary Microbiol. 114 269-274.

Gay, N.J., Gangloff, M., 2007. Structure and function of Toll receptors and their ligands Annu. Rev. Biochem. 76, 141-165.

Genersch, E., 2010. Honey bee pathology: current threats to honey bees and beekeeping. Appl. Microbiol. Biotechnol. 87, 87-97.

Gregory, P.G., Evans, J.D., Rinderer, T., de Guzman, L., 2005. Conditional immune-gene suppression of honeybees parasitized by Varroa mites. J. Insect Sci. 5, 7.

Gwak, G.-Y., Yoon, J.-H., Kim, K.M., Lee, H.-S., Chung, J.W., Gores, G.J., 2005. Hypoxia stimulates proliferation of human hepatoma cells through the induction of hexokinase II expression. J. Hepatol. 42, 358-364.

Henry, M., Beguin, M., Requier, F., Rollin, O., Odoux, J.-F., Aupinel, P., Aptel, J., Tchamitchian, S., Decourtye, A., 2012. A common pesticide decreases foraging success and survival in honey bees. Science 336, 348-350.

Hu, H., Bienefeld, K., Wegener, J., Zautke, F., Hao, Y., Feng, M., Han, B., Fang, Y., Wubie, A.J., Li, J., 2016. Proteome analysis of the hemolymph, mushroom body, and antenna provides novel insight into honeybee resistance against Varroa infestation. J، Proteome Res. 15, 2841-2854.

Inohara, N., Nuñez, G., 2002. ML-a conserved domain involved in innate immunity and lipid metabolism. Trends Biochem. Sci. 27, 219-221.

Kanbar, G., Engels, W., 2004. Visualisation by vital staining with trypan blue of wounds punctured by Varroa destructor mites in pupae of the honey bee (Apis mellifera). Apidologie 35, 25-30.

Kanost, M.R., 2013. Seine protease inhibiors from the serpin gene family in Manduca sexta and Drosophila melanogaster. Mol. Insect Sci. 139-146.

Khongphinitbunjong, K., de Guzman, L.I., Tarver, M.R., Rinderer, T.E., Chen, Y., Chantawannakul, P., 2015. Differential viral levels and immune gene expression in three stocks of Apis mellifera induced by different numbers of Varroa destructor. J. Insect Physiol. 72, 28-34.

Koleoglu, G., Goodwin, P.H., Reyes-Quintana, M., Hamiduzzaman, M.M., Guzman-Novoa, E., 2017. Effect of Varroa destructor, Wounding and Varroa Homogenate on Gene Expression in Brood and Adult Honey Bees. PloS one 12, e0169669.

Kuster, R.D., Boncristiani, H.F., Rueppell, O., 2014. Immunogene and viral transcript dynamics during parasitic Varroa destructor mite infection of developing honey bee (Apis mellifera) pupae. J. Exp. Biol. 217, 1710-1718.

Lansdown, A.B., 2002. Calcium: a potential central regulator in wound healing in the skin. Wound Repair Regeneration 10, 271-285.

le Conte, Y., Ellis, M., Ritter, W., 2010. Varroa mites and honey bee health: can Varroa explain part of the colony losses? Apidologie 41, 353-363.

Levin, S., Sela, N., Chejanovsky, N., 2016. Two novel viruses associated with the Apis mellifera pathogenic mite Varroa destructor. Sci. Rep. 6, 37710.

Liu, K., Dong, Y., Huang, Y., Rasgon, J.L., Agre, P., 2013. Impact of trehalose transporter knockdown on Anopheles gambiae stress adaptation and susceptibility to Plasmodium falciparum infection. Proc. Natl. Acad. Sci. 110, 17504-17509.

Ludvigsen, M., Jacobsen, C., Maunsbach, A.B., Honoré, B., 2009. Identification and characterization of novel ERC-55 interacting proteins: evidence for the existence of several ERC-55 splicing variants; including the cytosolic ERC-55-C. Proteomics 9, $5267-5287$. 
Marcos-Contreras, O., Ganguly, K., Yamamoto, A., Shlansky-Goldberg, R., Cines, D., Muzykantov, V., Murciano, J.-C., 2013. Clot penetration and retention by plasminogen activators promote fibrinolysis. Biochem. Pharmacol. 85, 216-222.

Michalski, A., Damoc, E., Hauschild, J.P., Lange, O., Wieghaus, A., Makarov, A., Nagaraj, N., Cox, J., Mann, M., Horning, S., 2011. Mass spectrometry-based proteomics using Q Exactive, a high-performance benchtop quadrupole Orbitrap mass spectrometer. Mol. Cell. Proteomics 10 (9), M111-011015.

Montagne, J., Stewart, M.J., Stocker, H., Hafen, E., Kozma, S.C., Thomas, G., 1999. Drosophila S6 kinase: a regulator of cell size. Science 285, 2126-2129.

Naora, H., Naora, H., 1999. Involvement of ribosomal proteins in regulating cell growth and apoptosis: translational modulation or recruitment for extraribosomal activity? Immunol. Cell Biol. 77, 197-205.

Narciso, C., Wu, Q., Brodskiy, P., Garston, G., Baker, R., Fletcher, A., Zartman, J., 2015. Patterning of wound-induced intercellular $\mathrm{Ca} 2+$ flashes in a developing epithelium. Phys. Biol. 12, 056005.

Navajas, M., Migeon, A., Alaux, C., Martin-Magniette, M.-L., Robinson, G., Evans, J., CrosArteil, S., Crauser, D., le Conte, Y., 2008. Differential gene expression of the honey bee Apis mellifera associated with Varroa destructor infection. Bmc Genomics 9, 301.

Parker, R., Guarna, M.M., Melathopoulos, A.P., Moon, K.-M., White, R., Huxter, E. Pernal, S.F., Foster, L.J., 2012. Correlation of proteome-wide changes with social immunity behaviors provides insight into resistance to the parasitic mite, Varroa destructor, in the honey bee (Apis mellifera). Genome Biol. 13, 1.

Qi, Y., Fan, P., Hao, Y., Han, B, Fang, Y., Feng, M., Cui, Z., Li, J, 2015. Phosphoproteomic analysis of protein phosphorylation networks in the hypopharyngeal gland of honeybee workers (Apis mellifera ligustica). J. Proteome Res. 14, 4647-4661.

Restrepo, S., Basler, K. 2016. Drosophila wing imaginal discs respond to mechanical injury via slow InsP3R-mediated intercellular calcium waves. Nat. Commun. 7.

Richards, E., Jones, B., Bowman, A., 2011. Salivary secretions from the honeybee mite Varroa destructor: effects on insect haemocytes and preliminary biochemical characterization. Parasitology 138, 602-608.

Robey, R.A., Hay, N., 2006. Mitochondrial hexokinases, novel mediators of the antiapoptotic effects of growth factors and Akt. Oncogene 25, 4683-4696.

Stanley-Samuelson, D.W., Jurenka, R.A., Cripps, C., Blomquist, G.J., de Renobales, M., 1988. Fatty acids in insects: composition, metabolism, and biological significance. Arch. Insect Biochem. Physiol. 9, 1-33.

Stewart, M.J., Denell, R., 1993. Mutations in the Drosophila gene encoding ribosomal protein S6 cause tissue overgrowth. Mol. Cellular Biol. 13, 2524-2535.

Strukova, S., 2006. Blood coagulation-dependent inflammation. Coagulation-dependent inflammation and inflammation-dependent thrombosis. Front. Biosci. 11, 59-80.

Surlis, C., Carolan, J.C., Coffey, M.F., Kavanagh, K., 2016. Proteomic analysis of Bayvarol $^{\circledast}$ resistance mechanisms in the honey bee parasite Varroa destructor. J. Apic. Res. 55, 49-64.

Theopold, U., Li, D., Fabbri, M., Scherfer, C., Schmidt, O., 2002. The coagulation of insect hemolymph. Cellular Mol. Life Sci. CMLS 59, 363-372.

Thompson, S., 1982. Effects of parasitization by the insect parasite Hyposoter exiguae on the growth, development and physiology of its host Trichoplusia ni. Parasitology 84, 491-510.

Thompson, S., Redak, R., Wang, L.-W., 2005. Nutrition interacts with parasitism to influence growth and physiology of the insect Manduca sexta L. J. Exp. Biol. 208, 611-623.

Tomoda, H., Igarashi, K., Cyong, J.-C., Omura, S., 1991. Evidence for an essential role of long chain acyl-CoA synthetase in animal cell proliferation. Inhibition of long chain acyl-CoA synthetase by triacsins caused inhibition of Raji cell proliferation. J. Biol. Chem. 266, 4214-4219.

van Vaerenbergh, M., Debyser, G., Smagghe, G., Devreese, B., de Graaf, D.C., 2015. Unraveling the venom proteome of the bumblebee (Bombus terrestris) by integrating a combinatorial peptide ligand library approach with FT-ICR MS. Toxicon 102, 81-88.

Vinson, S.B., Iwantsch, G., 1980. Host regulation by insect parasitoids. Quarterly Rev. Biol. 143-165.

Vorum, H., Jacobsen, C., Honoré, B., 2000. Calumenin interacts with serum amyloid P component. FEBS Lett. 465, 129-134.

Whitten, M.M., Tew, I.F., Lee, B.L., Ratcliffe, N.A., 2004. A novel role for an insect apolipoprotein (apolipophorin III) in $\beta$-1, 3-glucan pattern recognition and cellular encapsulation reactions. J. Immunol. 172, 2177-2185.

Wilfert, L., Long, G., Leggett, H., Schmid-Hempel, P., Butlin, R., Martin, S., Boots, M., 2016. Deformed wing virus is a recent global epidemic in honeybees driven by Varroa mites. Science 351, 594-597.

Williams, G.R., Tarpy, D.R., Vanengelsdorp, D., Chauzat, M.P., Cox-Foster, D.L., Delaplane, K.S., Neumann, P., Pettis, J.S., Rogers, R.E., Shutler, D., 2010. Colony collapse disorder in context. Bioessays 32, 845-846.

Zakar, E., Jávor, A., Kusza, S., 2014. Genetic bases of tolerance to Varroa destructor in honey bees (Apis mellifera L.). Insectes sociaux 61, 207-215.

Zera, A.J., Harshman, L.G., 2001. The physiology of life history trade-offs in animals. Annu. Rev. Ecol. Systematics 95-126.

Zhang, Y., Rai, M., Wang, C., Gonzalez, C. \& Wang, H. 2016. Prefoldin and Pins synergistically regulate asymmetric division and suppress dedifferentiation. Sci. Rep. 6 . 